\title{
RECOMBINATION OF IONS IN THE AFTERGLOW OF A CESIUM DISCHARGE
}

\author{
By Fred L. Mohler
}

\section{ABSTRACT}

A diseharge through a $500-\mathrm{cm}^{3}$ bulb is short-circuited by a motor-driven commutator and the radiation and electrical characteristics of the afterglow are studied. Radiation at a definite time interval after the cutoff was observed by means of a sectored disk on the commutator shaft, while electrical measurements were made by closing a probe circuit momentarily by means of a second commutator.

The afterglow fades at a rate which decreases with increasing pressure up to $46 \mu$ and remains nearly constant (the half value time for a 6-amp. discharge is $1.5 \times 10^{-3} \mathrm{sec}$ ) in the pressure range 46 to $110 \mu$. The spectral-intensity distribution of the continuous recombination spectrum indicates a much-lower electron temperature than in the discharge, decreasing with time from $1,360^{\circ} \mathrm{K}$ at $0.9 \times 10^{-3}$ sec to $1,200^{\circ} \mathrm{K}$ at $1.7 \times 10^{-3}$ sec.

The number of ions at any time during the afterglow is measured by the positiveion current to a negative probe, and the flow of ions to the wall is measured by the current to a collector against the bulb wall. The difference between the change in number of ions in the bulb and the flow of ions to the wall gives the number recombining in the space. From this is derived a recombination coefficient of $3.4 \times 10^{-10}$ in the pressure range 10 to $30 \mu$.

\section{CONTENTS}

I. Introduction ........

II. Apparatus and methods

III. Visual-intensity measurements _. _ _

IV. Spectrophotometric measurements _ _

V. Electrical measurements _...

VI. Discussion

\section{INTRODUCTION}

The rate of recombination of positive and negative ions in air and the common gases at pressures of the order of 1 atmosphere is known from many experiments. ${ }^{1}$ For the usual case where the number of positive and negative ions is equal,

$$
d n / d t=-\alpha n^{2},
$$

where $n$ is the number of ions of either sign per cubic centimeter and $\alpha$ is the recombination coefficient. For the common gases at a pressure of 1 atmosphere, $\alpha$ is of the order of $10^{-6}$. It decreases with decreasing pressure, probably in proportion to the pressure, though there is conflicting evidence on this point. The evidence supports Thomson's

\footnotetext{
${ }^{1}$ Loeb, Kinetic Theory of Gases, p. 583 (McGraw-Hill Book Co., New York, N. Y., 1934), gives a good summary on the subject.
} 
theory that recombination is the result of a three-body collision between ions of molecular mass and a neutral molecule. The usual experimental technique is not applicable at pressures much less than 0.1 atmosphere, but it is to be expected that with decreasing pressure the three-body process will become negligible and two-body recombination of positive ions and electrons with emission of radiation will become predominant. The observation of a continuous spectrum extending from a series limit to shorter wave lengths gives evidence for the existence of spontaneous two-body recombination. Boeckner and the author have measured the rate of recombination into a few quantum states by measuring the number of quanta of radiation emitted. ${ }^{2}$ In this way it was found that the recombination coefficient for recombination into the $6 \mathrm{P}$ state of cesium is $6 \times 10^{-14}$ for an electron temperature of $3,500^{\circ} \mathrm{K}$. The method gives no indication of the total recombination into all possible quantized states. There are also difficulties in evaluating theoretically the contribution of all levels for recombination with electrons with a temperature distribution.

The only important contribution on the rate of recombination in a low-pressure discharge is a study by Kenty of the decrease in electron concentration in the afterglow of an argon discharge. ${ }^{3}$ By means of a double commutator he measured by a probe wire the number of electrons per cubic centimeter at various time intervals after the discharge was cut off. Assuming that diffusion to the walls was negligible, he obtained a value of $\alpha=2 \times 10^{-10}$ (with a fivefold uncertainty, because of difficulties with the probe measurements) for an electron temperature of $3,100^{\circ} \mathrm{K}$ and a gas pressure of $0.8 \mathrm{~mm}$. His paper includes qualitative evidence that the loss of ions by wall diffusion is small. Miss Hayner ${ }^{4}$ had previously shown that the plasma condition which exists in the discharge persists after the discharge is cut off. That is the space maintains a positive charge with respect to the walls so that the rate at which electrons reach the walls is limited to the rate at which the heavy positive ions can diffuse. Because of this phenomenon the study of recombination at low pressures is practicable.

As the rate of recombination is a fundamental atomic constant, the author has tried to eliminate some of the uncertainties in previous work. The cesium discharge has been used and this study includes visual photometric measurements of the rate of decay of the afterglow, some spectrophotometric measurements, and probe measurements of the change in electron concentration and diffusion of ions to the walls. The visual measurements were an essential preliminary to see under what range of conditions the afterglow could be considered as a uniformly ionized volume.

\section{APPARATUS AND METHODS}

To reduce the effect of ion diffusion to the walls it is desirable to use a large bulb, though the size will be limited by the discharge current available. In most of this work the discharge tube has been a $500-\mathrm{cm}^{3}$ bulb with cathode and anode in diametrically opposite side tubes. The discharge was operated on direct current and interrupted by a commutator running at a speed of about $1,200 \mathrm{rpm}$. There are

${ }^{2}$ F. L. Mohler and C. Boeckner, BS J. Research 2, 489 (1929) RP46. F. L. Mohler, BS J. Research 10, 771 (1933) RP565 and correction noted in J. Research NBS 17, 849 (1936) RP948.

3 Kenty, Phys. Rev. 32, 624 (1928).

4 Hayner, Z. Phys. 35, 365 (1925). 
obvious advantages in short-circuiting the discharge rather than breaking the circuit, as the instant of cutoff is then perfectly definite and all sparking occurs at the time when the discharge comes on. The cesium discharge operates at about $10 \mathrm{v}$, and by running the discharge on a $100-\mathrm{v}$ battery with series resistance, a discharge of $6 \mathrm{amp}$ could be interrupted without destructive arcing.

The commutators were copper segments set flush in the edge of a fiber disk and contact was made by carbon brushes. For the intensity measurements there were four segments on the commutator and a disk with four 1-cm sectors cut in it was mounted on the same shaft as the commutator to permit viewing the afterglow at known time intervals after the cutoff. A long slit immediately in front of the discharge was focused on the plane of the sectored disk, and by looking through the disk the afterglow was viewed stroboscopically at a time after the cutoff determined by the angular separation between the sector and the image at the instant of cutoff, and the motor speed. Each flash lasted $0.0007 \mathrm{sec}$ at $1,200 \mathrm{rpm}$. The intensity was measured by a simple photometer. A white surface extending into the image of the discharge was illuminated by a headlight lamp with a yellow filter in front of it and intensity was matched by changing the distance of the lamp and by interposing neutral screens.

To photograph the spectrum of the afterglow the stroboscopic image was focused on the slit of a spectrograph. As a basis for intensity measurements, there was included on each plate a series of exposures to a calibrated strip lamp placed back of the sectored disk.

For the electrical measurements, two commutators with two segments on each were used. One of these short-circuited the discharge for about $0.004 \mathrm{sec}$ and the other put the probe or disk electrode into the circuit for about $0.001 \mathrm{sec}$ at an adjustable time interval after the cutoff. The probe electrodes consisted of a disk $3 \mathrm{~cm}$ in diameter against the wall of the bulb to measure the flow of ions to the wall and a wire $0.5 \mathrm{~mm}$ in diameter extending radially with an exposed surface $6 \mathrm{~cm}$ long. This was operated at a negative voltage to measure the positive-ion current. This current is proportional to the electron concentration at a given pressure, and the method of determining the proportionality factor is described later.

There is a small potential drop across the commutator when the current is short-circuited and this modifies somewhat the conditions in the afterglow. A low-resistance potential divider has been put in the discharge circuit to balance this potential difference.

\section{VISUAL INTENSITY MEASUREMENTS}

The appearance of the discharge undergoes an abrupt change at the instant of the cutoff and then fades relatively slowly. At low pressure the discharge is a purplish pink color which fills the bulb and the afterglow is pale yellow. With increasing pressure the color change becomes less marked but the intensity distribution changes. In the discharge the intensity becomes concentrated near the cathode and anode, while in the afterglow the glow is fairly uniform but brightest near the center of the bulb. At vapor pressures greater than $100 \mu$ of mercury the glow expands from the center during the afterglow period. The dark period described by Miss Hayner ${ }^{5}$ and

\footnotetext{
${ }^{6}$ Hayner, Z. Phys. 35, 365 (1925).
} 
Dr. Kenty ${ }^{6}$ is not conspicuous in the cesium discharge. In the spectrum of the afterglow the higher series lines and continuous bands beyond the series limits are relatively intense and the appearance supports the view of Miss Hayner and Dr. Kenty that the glow is predominantly a recombination spectrum. It is known that the electron temperature drops very rapidly after the cutoff, ${ }^{7}$ and it seems safe to assume that excitation drops essentially to zero in the short transition period before the uniform glow fills the bulb.

If it is assumed that the intensity, $j$, comes entirely from recombination, then $j$ is proportional to $n^{2}$, that is,

$$
n=k j^{1 / 2}
$$

where $k$ is an arbitrary constant which changes rather slowly with the electron temperature. With this limitation $j^{1 / 2}$ is a measure of the electron concentration without any assumption that the change in $n$ comes entirely from spontaneous recombination. For the case that the change comes entirely from recombination, an integration of equation 1 over the time interval $t_{1}$ to $t_{2}$ gives

$$
\frac{1}{n_{2}}-\frac{1}{n_{1}}=\alpha\left(t_{2}-t_{1}\right)
$$

and using intensity as a measure of electron concentration

$$
\frac{1}{j_{2}^{1 / 2}}-\frac{1}{j_{1}^{1 / 2}}=k \alpha\left(t_{2}-t_{1}\right)
$$

A plot of $j^{-1 / 2}$ versus time will then give a straight line if volume recombination is predominant, but because of wall recombination, the observed curves will in general be steeper and concave upwards.

Measurements of intensity as a function of time after the cutoff have been plotted on this scale to see if the time variation of intensity is consistent with the assumption that volume recombination is predominant. Figure 1 gives a typical group of measurements based on intensity at a time $t$ after cutting off a 5.8-ampere discharge. The curves start out nearly straight and curve upward at an increasing rate. With increasing pressure up to $21 \mu$ the slope near the origin rapidly decreases, presumably because of decreasing diffusion to the walls. In the pressure range 40 to $110 \mu$ there is a nearly constant minimum slope and at higher pressures the slope again increases. It is to be expected that $\alpha$ will increase with the pressure, but as it appears that the distribution of ionization is less uniform at high pressure, the evidence for a real change in $\alpha$ is uncertain. Since diffusion to the walls will decrease with increasing pressure, the results indicate that as pressures increase above $21 \mu$ volume recombination becomes predominant.

An approximate numerical value of the constant $k$ in equation 3 can be obtained with the assumption that the intercept at $t=0$ is proportional to $1 / n_{0}$, where $n_{0}$ is the number of electrons per cubic centimeter in the steady discharge. A similar bulb with a probe surface midway between the wall and the center of the bulb gave a

${ }^{6}$ Kenty, Phys. Rev. 32, 624 (1928).

7 Randall and Webb, Phys. Rev. 48, 544 (1935). 
mean value $n_{0}=1.9 \times 10^{12}$ in the pressure range 46 to $110 \mu .^{8}$ The slope of the curve of figure 1 obtained in this pressure range gives a value $\alpha=3.5 \times 10^{-10}$ on the assumption that diffusion to the walls is negligible.

\section{SPECTROPHOTOMETRIC MEASUREMENTS}

The spectrum of the cesium afterglow has been briefly described by Boeckner and the author. ${ }^{9}$ At a pressure of $100 \mu$ the line-intensity distribution is much like that of the discharge, but the continuous spectrum decreases beyond the series limit much faster than in the

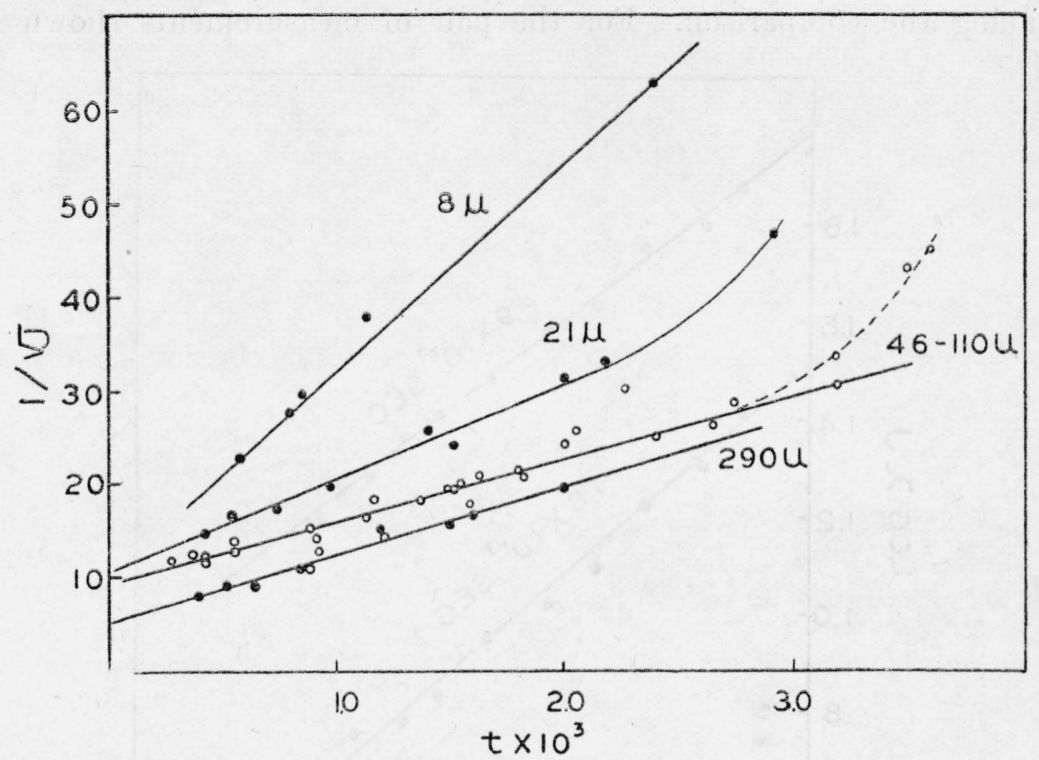

FIGURE 1.-Intensity, $j$, of the afterglow as a function of time after the cutoff on a scale of $1 / j^{1 / 2}$ in arbitrary units versus time.

Vapor pressure in microns of mercury is indicated on each curve.

discharge. This indicates that the electron temperature is much lower in the afterglow and the measurement of the intensity distribution offers a good method of measuring the instantaneous values of the electron temperature in the afterglow. The intensities at two wave lengths, $\lambda_{1}$ and $\lambda_{2}$, at intervals $V_{1}$ and $V_{2}$, in electron volts beyond the series limits are related to the electron temperature by the equation ${ }^{10}$

$$
T_{e}=5040 \frac{V_{2}-V_{1}}{\log \lambda_{1} j_{1}-\log \lambda_{2} j_{2}}
$$

Figure 2 gives a plot based on measurements of the band beyond the $6 P$ limit in the wave-length range 5000 to $4600 \mathrm{~A}$. Measurements were made at time intervals of $0.90 \times 10^{-3} \mathrm{sec}$ and $1.80 \times 10^{-3} \mathrm{sec}$

8 This figure for $n_{0}$ involves a large and rather uncertain correction factor for the effect of vapor pressure on the probe current. Mohler, J. Research NBS 17, 849 (1936) RP948.

9 F. L. Mohler and C. Boeckner, BS J. Research 2, 489 (1929) RP46.

10 F. L. Mohler, BS J. Research 10, 771 (1933) RP565. 
after short-circuiting a 5.8-amp discharge at a pressure of $68 \mu$. The slopes of the curves leadto values of 1,350 and $1,200^{\circ} \mathrm{K}$ for the electron temperatures at these two times. The electron temperature during the discharge is about $2,700^{\circ} \mathrm{K}$. A second experiment at a pressure of $85 \mu$ gave temperatures of 1,360 and $1,200^{\circ} \mathrm{K}$ at intervals of $0.87 \times 10^{-3}$ and $1.63 \times 10^{-3}$ sec after the cutoff.

It has been attempted to compare the absolute intensities at the limit with the recombination spectrum of the cesium positive column at low pressure ${ }^{11}$ as a basis for measuring the number of electrons per cubic centimeter in the afterglow.

It is assumed that the intensity at the limit is proportional to $T_{e}^{-3 / 2}$. There are experimental uncertainties of perhaps 50 percent in making the comparison. For the pair of measurements shown in

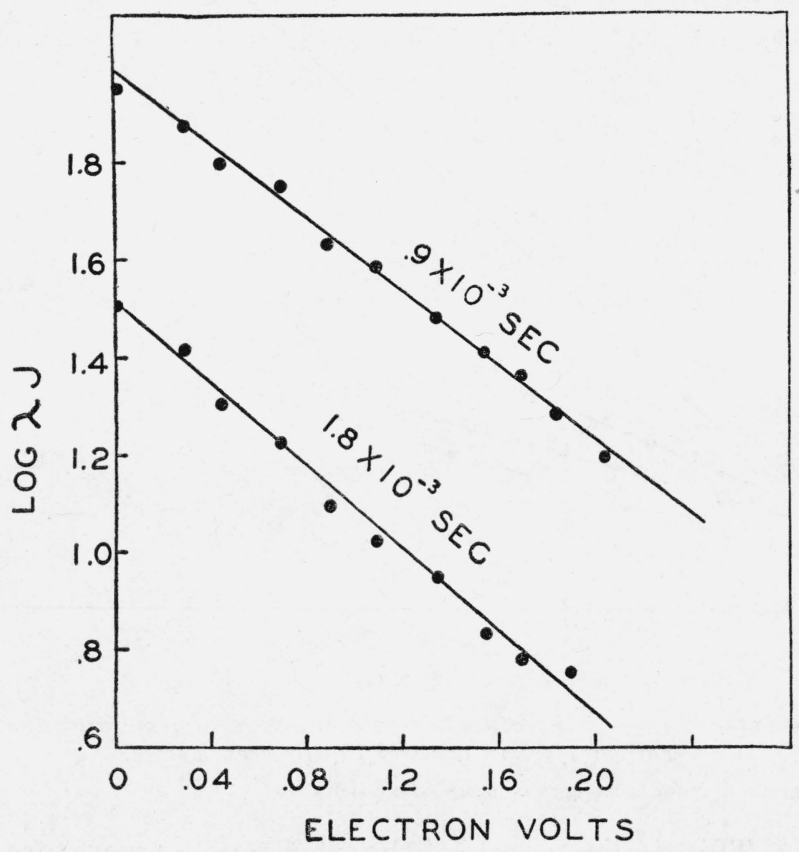

FIGURE 2.-Semilogarithmic plot of intensity distribution in the continuous spectrum of the afterglow beyond the $6 \mathrm{P}$ limit on a scale to give electron temperature.

Ordinates give $\log \lambda j$ in arbitrary units and abscissas give the frequency interval beyond the series limit, in units of electron volts. The slopes give electron temperatures of 1,350 and $1,200^{\circ} \mathrm{K}$ for the upper and lower lines, respectively.

figure $2, n_{e}=1.78 \times 10^{12}$ and $0.95 \times 10^{12}$ for the second pair referred to above $n_{e}=1.45 \times 10^{12}$ and $0.98 \times 10^{12}$. Again assuming that wall recombination is negligible, these values in equation 2 give values of

$$
\alpha=5.5 \times 10^{-10} \text { and } 4.3 \times 10^{-10}
$$

\section{ELECTRICAL MEASUREMENTS}

The change in the total number of ions $\Delta N$ in the bulb in any interval $\Delta t$ after the cutoff is equal to the number of ions flowing to the wall, plus the number recombining in the space. This assumes, as seems

11 F. L. Mohler, BS J. Research 10, 771 (1933) RP565. 
reasonable, that there is no production of ions in the afterglow period. With the approximation that the distribution of ions is uniform and that the current density to the walls is uniform

$$
\frac{\Delta N}{\Delta t}=\frac{4 \pi r^{2} i_{v 0}}{e}+\frac{4}{3} \pi r^{3} \alpha n^{2},
$$

where $r$ is the radius of the bulb, $i_{w} / e$ the number of ions flowing to the wall per unit area, and $\alpha n^{2}$ the number recombining per unit volume. The current density of positive ions to the wall is found by measuring the ion current to the disk at negative potentials and extrapolating to the potential of zero current.

The conventional method of measuring electron concentration is to measure the electron current to a small positive probe, but difficulties

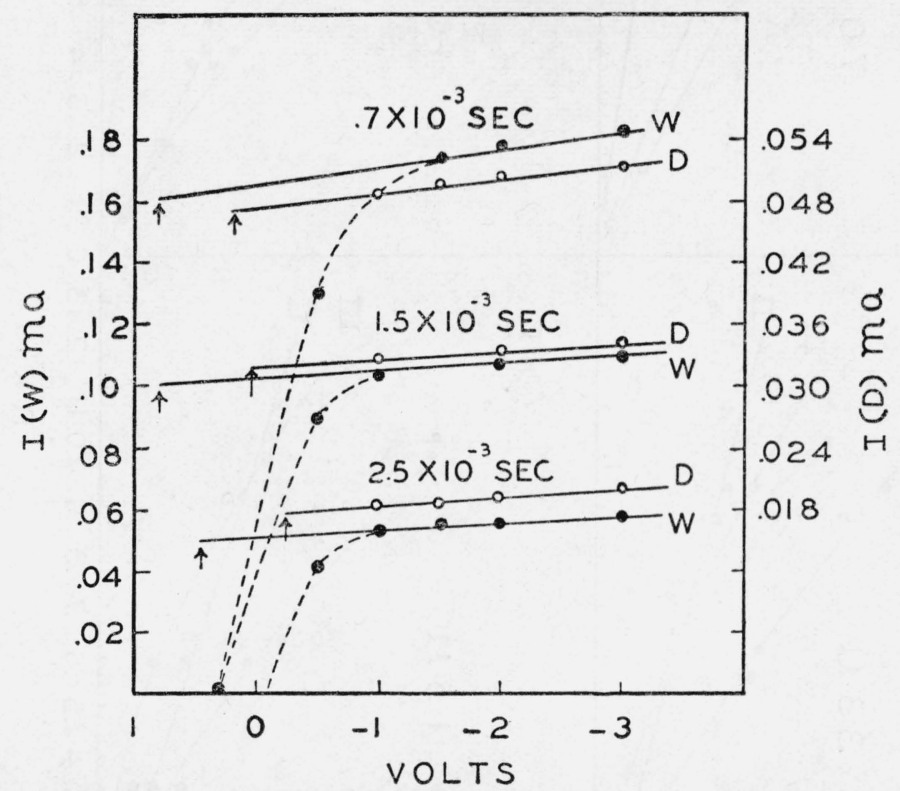

Figure 3.-Current-voltage curves for the negative wire, $W$, and disk, $D$, at various times after the cutoff.

Currents are plotted on two different scales.

were encountered in obtaining reproducible results and the method was adopted of measuring the positive current to a large negative probe. This gives very good precision, but it is essential to find the proportionality factor between ion current and ion concentration. This factor was found from the condition that in equation 5 at low currents the term on the right is negligible. That is, by setting $\Delta N=k i_{+}$the factor $k$ is found which satisfies the equation. The method is not very accurate, but it happens that for the higher discharge currents the value of $\alpha$ is quite insensitive to the absolute value of $n$. As the use of equation 5 involves double differences it is more important to have precise relative values.

Figure 3 gives typical current-voltage curves at various time intervals after cutting off a 6 -amp discharge in cesium at a pressure 
of $13 \mu$. Curves $D$ give the plate current and curves $W$ the current to the wire probe. The figure indicates how the curves are extrapolated to the space potential and wall potential for the wire and disk, respectively. The slope of the plate current-voltage curve comes from a capacity effect. The change in the current to the wire

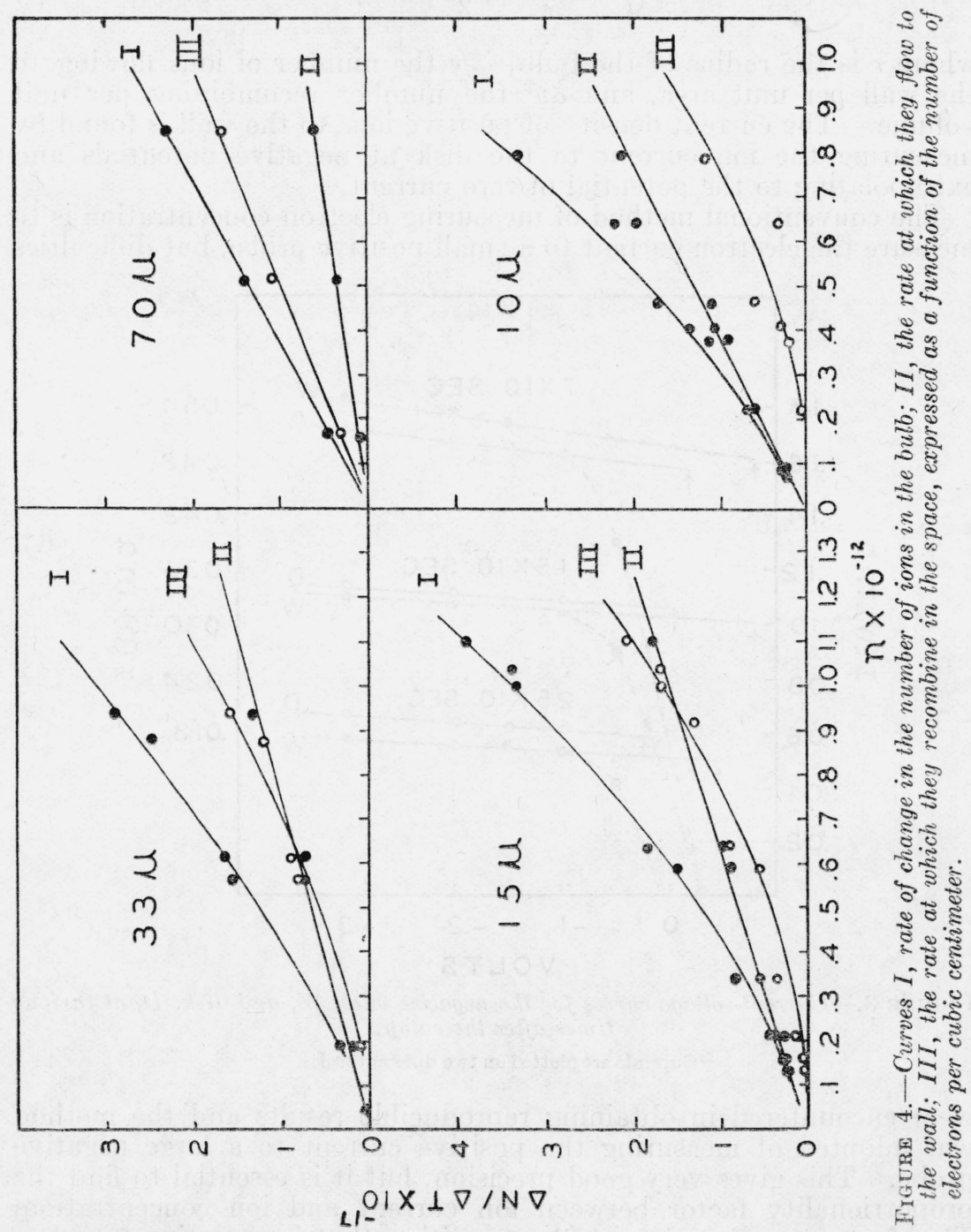

can be accounted for by the change in area of the space-charge sheath. In some cases the space-charge equation has been used to compute the current at the space potential, but the simple linear extrapolation gives nearly the same result. It is evident that the time change in the probe current is slightly greater than that in the plate 
current, which shows that the concentration of ions changes faster at the center than at the wall. With the proportionality factor between ion current and ion concentration found from similar low-current measurements, the data of figure 3 give $\Delta N / \Delta t$ and $i_{w}$ at various times. In figure 4, curves $I, \Delta N / \Delta t$ is plotted as a function of the mean value of $n$ over the time interval $\Delta t$ for four different pressures. Each curve gives values obtained with different values of the current for the time interval $1.5 \times 10^{-3}$ to $2.5 \times 10^{-3}$ sec. after the cutoff.

Curves $I I$ give the mean values of the wall current in ions per second for the same conditions. At each pressure it is a nearly linear function of $n$ and it decreases with increasing pressure. For small values of $n$, curve $I I$ approaches curve $I$, that is, the wall current accounts for nearly the entire change in the number of ions. Curves $I I I$ give the difference between curves $I$ and $I I$, which is the space recombination in the total volume of the bulb. At the higher pressures the space recombination is greater than the wall recombination, except at very low values of $n$. The precision seems to be best at $15 \mu$ and here the space recombination is within experimental error proportional to $n^{2}$, as is to be expected on theoretical considerations. At higher pressures, curve III has less curvature than is to be expected. A value of $\alpha=3.4 \times 10^{-10}$ fits all observations at pressures of $30 \mu$ or less, within the range of experimental uncertainty. At $70 \mu$ the measurement at $n=8.5 \times 10^{11}$ gives a value of $4 \times 10^{-10}$, while lower currents give higher values.

Measurements over the time interval 0.7 to $1.5 \times 10^{-3} \mathrm{sec}$ lead to similar values of $\alpha$ but with a considerably larger experimental uncertainty. Here the first contact comes very soon after the cutoff, probably during the transition period preceding the uniform afterglow, and this may well account for accidental fluctuations in the measurements.

The best value of the recombination coefficient for pressures of $30 \mu$ or less is

$$
\alpha=(3.4 \pm .5) \times 10^{-10}
$$

This value is consistent with the less-accurate estimates obtained in the pressure range 46 to $110 \mu$ by optical methods. Volume recombination accounts for 0.6 or 0.7 of the total change in $N$ under the conditions of the optical measurements, and the values of $\alpha$ derived from them must be multiplied by this factor to correct for wall loss. The spectrophotometric measurements give a corrected value of

$$
\alpha=3.6 \times 10^{-10} .
$$

\section{DISCUSSION}

The value of the recombination coefficient found here agrees with the value obtained by Kenty in the argon discharge well within his estimated range of uncertainty. The value of $3.4 \times 10^{-10}$ was found for an electron temperature of about $1,200^{\circ} \mathrm{K}$. The value probably varies inversely as the first or a higher power of the temperature, and a value of less than $2 \times 10^{-10}$ is to be expected in the cesium discharge. The relative importance of volume recombination in a columnar discharge can be estimated on this basis. For a 4-amp current at a pressure of $15 \mu$ in a tube $1.8 \mathrm{~cm}$ in diameter, the flow of ions to the 
walls is $10^{18}$ ions per second per centimeter of tube, while the volume recombination is less than $1 . \times 10^{17}$. At $290 \mu$ the flow to the walls is about $5 \times 10^{17}$ and the volume recombination nearly an equal amount. It is concluded that volume recombination in a columnar discharge is certainly negligible at the lower pressures. Whether or not it becomes important between 100 and $300 \mu$ cannot be asserted definitely until more is known concerning the pressure and temperature variation of the recombination coefficient.

Washington, July 9, 1937. 